\title{
Article \\ A Probabilistic-Based Deep Learning Model for Skin Lesion Segmentation
}

\author{
Adekanmi Adeyinka Adegun ${ }^{1}\left(\mathbb{D}\right.$, Serestina Viriri ${ }^{1, * \mathbb{C}}$ and Muhammad Haroon Yousaf ${ }^{2} \mathbb{C}$ \\ 1 Computer Science Discipline, University of KwaZulu-Natal, Durban 4000, South Africa; \\ 218082884@stu.ukzn.ac.za \\ 2 Department of Computer Engineering, University of Engineering and Technology, Taxila 47050, Pakistan; \\ haroon.yousaf@uettaxila.edu.pk \\ * Correspondence: viriris@ukzn.ac.za
}

check for updates

Citation: Adegun, A.A; Viriri, S.; Yousaf, M.H. A Probabilistic-Based Deep Learning Model for Skin Lesion Segmentation. Appl. Sci. 2021, 11, 3025. https://doi.org/10.3390/ app11073025

Received: 11 November 2020 Accepted: 5 January 2021 Published: 29 March 2021

Publisher's Note: MDPI stays neutral with regard to jurisdictional clai$\mathrm{ms}$ in published maps and institutional affiliations.

Copyright: (C) 2021 by the authors. Licensee MDPI, Basel, Switzerland. This article is an open access article distributed under the terms and conditions of the Creative Commons Attribution (CC BY) license (https:// creativecommons.org/licenses/by/ $4.0 /)$.

\begin{abstract}
The analysis and detection of skin cancer diseases from skin lesion have always been tedious when done manually. The complex nature of skin lesion images is one of the key reasons for this. The skin lesion images contain noise and artifacts such as hairs, oil and bubbles, blood vessels, and skin lines. They also have variegated colors, low contrast, and irregular borders. Various computational approaches have been designed in the past for aiding in the detection and diagnosis of skin cancer diseases using skin lesion images. The existing techniques have been limited due to the interference of the aforementioned features of skin lesion. Recently, machine learning techniques, in particular the deep learning techniques have been used for the detection of skin cancer. However, they are still limited to the fuzzy and irregular borders of skin lesion images coupled with the low contrast that exists between the diseased lesion and healthy tissues. In this paper, we utilized a probabilistic model for the enhancement of a fully convolutional network-based deep learning system to analyze and segment skin lesion images. The probabilistic model employs an efficient mean-field approximate probabilistic inference approach with a fully connected conditional random field that utilizes a Gaussian kernel. The probabilistic model further performs a refinement of skin lesion borders. The whole framework is tested and evaluated on publicly available skin lesion image datasets of ISBI 2017 and PH2. The system achieved a better performance, having an accuracy of $98 \%$.
\end{abstract}

Keywords: fully convolutional networks; Gaussian kernel; conditional random fields; probabilistic model; skin cancer; segmentation

\section{Introduction \\ 1.1. Background}

Skin cancer is generally caused by the abnormal growth of skin cells. This can cause unrepaired DNA damage and lead to multiple mutations [1,2]. This can lead to a rapid multiplication of abnormal cells that form malignant tumors. The main types of skin cancer are basal cell carcinoma (BCC), squamous cell carcinoma (SCC), and melanoma cancer [2]. Melanoma is the most deadly for skin cancer with a very high tendency of rapid malignancy if not discovered quickly. According to WHO (World Health Organization), the incidents of this type of cancer has been increasing over the past few decades with over 2 million cases of non-melanoma skin cancers [3].

The detection and diagnosis of skin cancer disease require an efficient framework for the analysis of skin lesion images to identify the Region of Interest (ROI) through segmentation. Segmentation is a process of identification and separation of the diseased skin lesion ROI from the normal skin tissues in order to identify a potential cancerous region. Various approaches have been employed to achieve this task in the past [4]. Traditional machine learning techniques have been utilized for the segmentation of skin lesion. Very recently, machine learning techniques have been employed for the analysis 
of medical images towards the detection and diagnosis of diseases. The segmentation algorithms [5-11] commonly used have been categorized into the following:

- Threshold-based Otsu's method and multi-level thresholding;

- Clustering such as Fuzzy C-Means (FCM) and K-Means clustering;

- Edge-based or region-based algorithms such as Region Adjacent Graph (RAG) merging algorithm, edge contour, watershed transform;

- Fused-based algorithms such as Discrete Wavelet Transform-based fusion, Principal component analysis (PCA)-based fusion;

- $\quad$ Active contour-based such as gradient vector flow (GVF);

- Soft computing Artificial Neural Network, Genetic Algorithms etc.

These methods have limitations due to their mode of operations [12] as they utilize different mathematical equations and functions to define how the observed features relate to a given diagnosis. They also rely on handcrafted features extracted using traditional feature extraction techniques which are very sensitive to noise and imprecise data such as skin lesion images. Very recently, deep learning techniques have been employed for the segmentation of skin lesions [13]. These techniques are generally more powerful than traditional computer aided design techniques such that they possess the ability to learn discriminative features [14]. However, their performances are still limited due to irregular and fuzzy boundaries of the skin lesions, as shown in Figure 1.

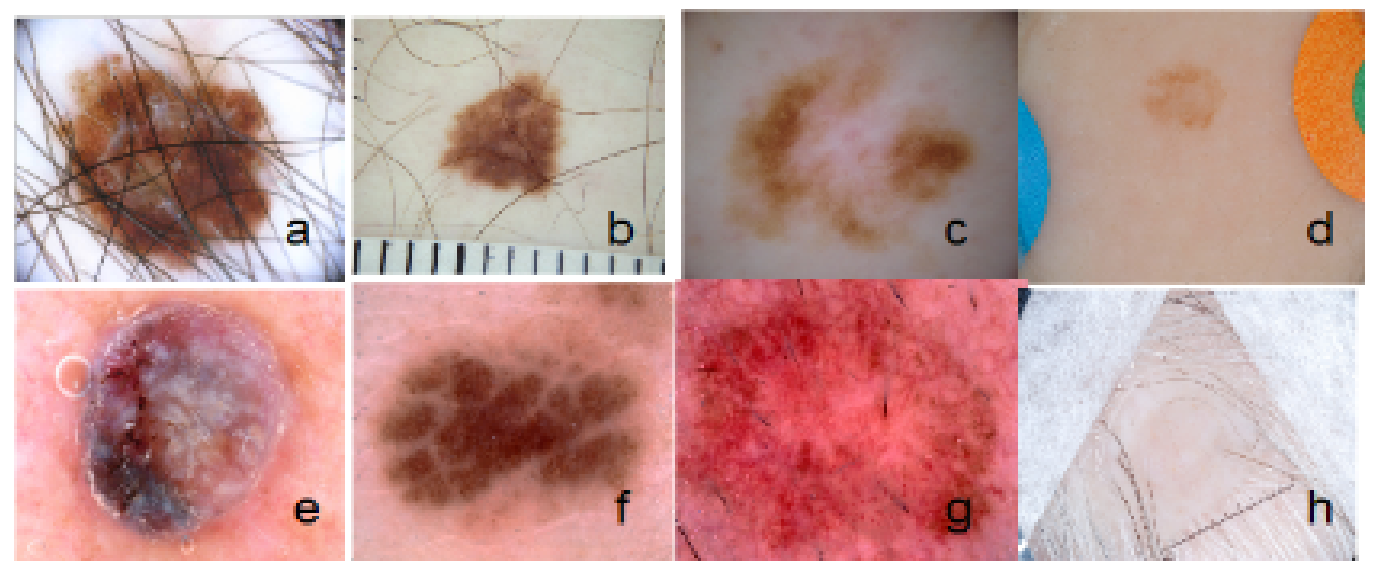

Figure 1. Skin lesions with challenging features such as hair artefact, ruler mark artefact, low contrast, bubbles, and irregular boundaries. (a) hair artefact; (b) ruler mark artefact; (c) low contrast; (d) color illumination; (e) bubbles; (f) irregular boundaries; (g) blood vessels; (h) frame artefact.

In order to proffer solutions to the aforementioned challenges, there is a need to integrate an efficient optimization model into deep learning to improve the performance of the deep learning system. In this paper, we employ a probabilistic-based deep learning model for skin lesion segmentation. The framework utilizes a fully connected Conditional Random Fields (CRFs) model with efficient mean-field approximate probabilistic inference for contour refinement and lesion boundaries localization. This ensures the selection of most discriminant deep features. The probabilistic-based deep learning model is combined with light-weight encoder-decoder architecture for efficient skin lesion segmentation.

It is evident that some recent enhancements in machine learning algorithms and dermoscopic techniques do not localize and segment the skin lesion accurately [15]. They also have a tendency of reducing the misclassification rate when not properly done. In this paper, an efficient framework has been proposed in which a deep learning model is rationally enhanced with a probabilistic construct. In this method, the segmented lesion contrast is enhanced through a probabilistic model that is constructed using Gaussian kernel, with an efficient mean field and approximate probabilistic inference for contour refinement and lesion boundaries localization. The proposed framework efficiently manages the computational resources and improves the diagnosis rate, thus speeding up detection 
and treatment by reducing the mortality rate of the disease. Skin cancer disease can be life-threatening and the timely detection of skin cancer can save lives.

\subsection{Related Works}

Various works have been done in the past for the enhancement of machine learning algorithms for a better performance and reduced misclassification rate through techniques such as probabilistic models, Gaussian, saliency techniques, etc. [16,17]. For example, Sigurdsson et al. [17] also developed a probabilistic feature extraction model with a feed forward neural network to classify the skin lesions. Barata et al. [18] employed two techniques of global features and local features using Bag of Features (BoF) to detect melanoma from dermoscopy images. A Bayesian framework was proposed to better delineate the shape and boundaries of skin lesion after saliency detection by Ahn et al. [19]. Bi et al. [20] proposed an automated method for skin lesion segmentation using a deep classspecific learning approach. The method combined probability-based step-wise integration for an improved segmentation output.

Khan et al. [16] proposed a strategy built on saliency valuation for the selection of most discriminant deep features selection. Their model was also enhanced using a Gaussian model. The most discriminant features are later subjected to a classification step. These techniques were used for the segmentation of the images that were later extracted by a CNN (Convolutional Neural Network) model. Jahanifar et al. [21] proposed a supervised saliency detection method based on Discriminative Regional Feature Integration (DRFI) for the segmentation of dermoscopic images. The method incorporated regional contrast, property, background descriptors, random forest regressor, and multi-level segmentation for an improved saliency detection method, mDRFI. The integration of deep learning methods with probabilistic models such as CRF and MRF(Markov Random Field) has been proven to be very effective in object detection. However, the implementation of such models on medical images, most especially skin lesion, is yet to be fully explored. Hence, this work aims at further exploring these models for the efficient segmentation of skin lesion images.

This work aims to develop a computationally less expensive system for robust and accurate skin lesion segmentation. In our proposed framework, a linear combination of Gaussian edge potentials with mean field approximation was applied to facilitate efficient inference and learning. This will eliminate the repeated inference with the back propagation training of the deep model and reduce computational cost. Our proposed architecture employs a reduced-size deep convolutional network that utilizes a dice loss function. The performance evaluation shows that the architecture gives significantly better segmentation results, most especially along lesion boundaries.

\subsection{Contributions}

The following are the main contributions of this research work:

- Modeling a light-weight deep learning framework that is composed of an encoderdecoder network limited to just five levels of network layers and with a minimized filter size for a lower consumption of computational resources;

- Integrating the deep learning model with a probabilistic model for further output enhancement through lesion border refinement by Gaussian kernel.

\section{Methods and Techniques}

This section explains the proposed framework in detail. The architectural diagram of the deep learning system and the integration with the probabilistic model is presented in Figures 2 and 3, respectively. 


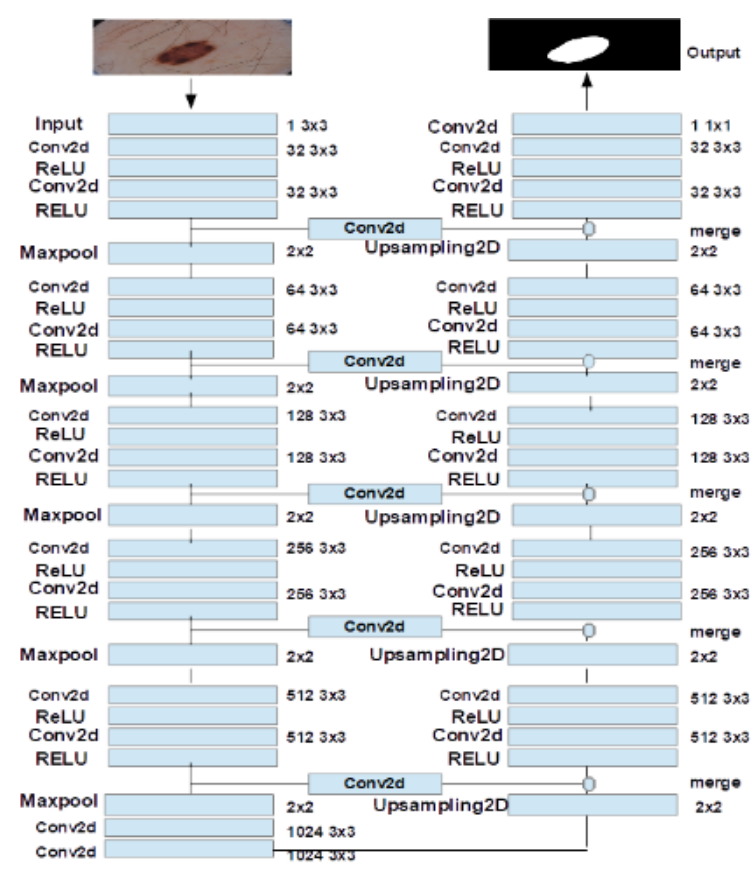

Figure 2. Layout diagram for a fully convolutional deep learning system.

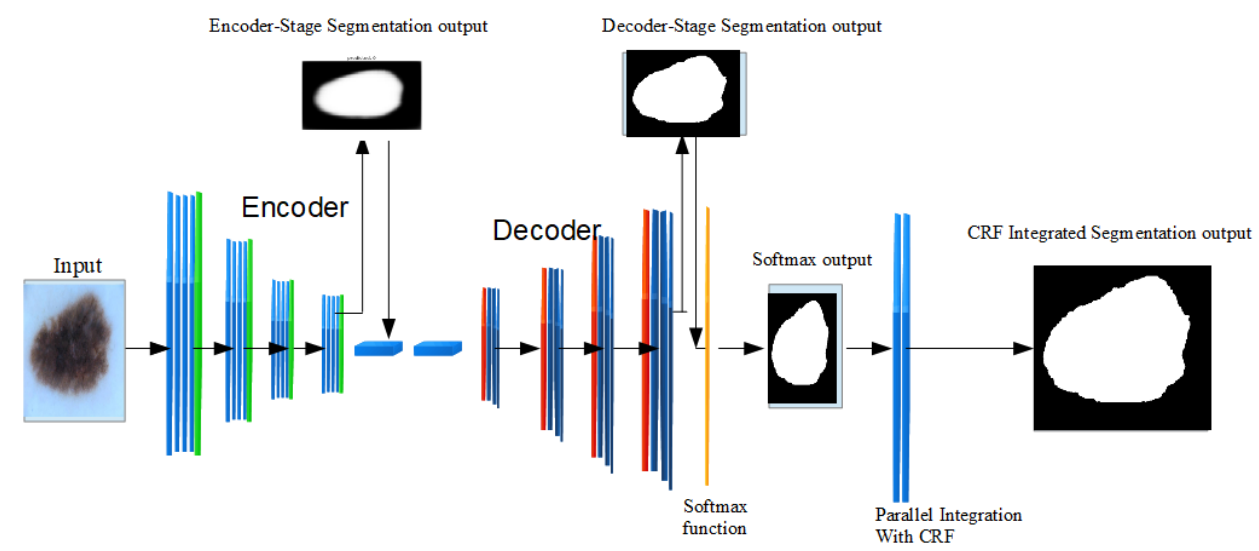

Figure 3. The proposed architectural diagram for a fully convolutional deep learning system enhanced with a probabilistic model.

\subsection{Image Pre-Processing and Data Augmentation}

\subsubsection{Image Size and Normalization}

A series of automated operations are first performed on the images to allow for efficient processing on the framework. We performed the pre-processing tasks to present all the images on the same scale and resolution through cropping and resizing. Further processing such as re-sampling was also performed on the images. The images were then normalized through pixel value mean and standard deviation computation for data centering and normalization.

\subsubsection{Image Augmentation}

The augmentation process adopts the on-the-fly approach for transformation and multiplication. These transformations took place with a random displacement and smoothening operation using the Gaussian filter. Other processes included setting horizontal flip as true, rotation as random, zoom range as 0.2 , and shear range as 0.2 on the images. 


\subsection{Deep Learning Model}

The framework employed a deep learning model [22] that utilized fully convolutional network architecture for the segmentation of skin lesion images. The proposed model is made up of a fully convolutional network composed of both the encoder network for features image up-sampling and a decoder network for the image down-sampling process. The decoder maps the low resolution discriminative features from the encoder to higher and full input resolution features to learn the complex and inhomogeneous skin lesion features. The architecture is an enhanced deeply-supervised encoder-decoder network where the encoder and decoder sub-networks are connected through a series of skip pathways.

The network employed in this system is enhanced by limiting the encoder-decoder level to five and also minimizes the filter size and number of feature maps as shown in Figure 2. The convolutional layer extracts the features of the input image as feature maps through the filters. The max-pooling layer performs down-sampling by reducing the sizes of the extracted feature maps. This helps in eliminating over-fitting and features redundancy and thereby minimizes computation time. The up-sampling layer recovers the reduced feature maps to its original size. The reduction of the spatial features resolution during the down-sampling process of the input image and the transit between the downsampling and up-sampling layers results in the missing features of some certain pixels.

\subsubsection{Encoder}

The encoder part is composed of five blocks with each block having two convolutional layers and one down-sampling layer. The convolutional layer utilizes the ReLu activation function. Convolutional network produces feature map through convolution process. The network consists of basic components such as filter kernels and the non-linear activation function ReLU. The activation function is applied on the feature map and the filter kernel as stated in Equation (1) below. This provides non-linearity to the network.

\subsubsection{Decoder}

The decoder part also consists of five blocks with each block having two convolutional layers and one up-sampling layer with a concatenation function. This concatenates the up-sampling layer input with the corresponding feature map from the convolutional layer in the encoder part.

\subsection{Probabilistic Model Integration}

The probability model is a conditional model that employs Gibbs distribution $[23,24]$ in which the probability $P(y \mid x)$ is modeled as:

$$
P(y \mid x)=\frac{1}{K(x)} \exp [-E(y, x)]
$$

where $X: x 1 \cdots x N$ are the input image, $Y: y 1 \cdots y N$, as the image mask, $E(x \mid y)$ is the cost of allotting label pixels to image pixels referred to as energy, and $\mathrm{K}$ is the partition function, which is stated as:

$$
K(x)=\sum_{y} \exp [-E(y, x)]
$$

In order to find the optimal estimation of $x$ given the observed image $Y$ using Maximum a posteriori (MAP), we have:

$$
x=\operatorname{argmax}_{x} P(x \mid Y) .
$$

The probabilistic model with each node representing a pixel in an image, $I$.

The unary potentials computes the cost of allotting the image masks $y$ to image pixels $x$ while the pair-wise computes the cost between neighboring pixels. 
This can be presented as:

$$
E(x)=\sum_{i} \psi\left(x_{i}\right)+\sum_{i<j} \psi\left(x_{i}, x_{j}\right)
$$

where $X: x 1 \cdots x N$ represents the input image, $Y: y 1 \cdots y N$ represents the labeling mask, $\Psi\left(x_{i}\right)$ represents the unary potentials, and $\Psi\left(x_{i}, x_{j}\right)$ represents the pair-wise potentials.

The unary potential identifies the probability of a pixel belonging to a certain class of image, such as foreground or background. The pair-wise potential identifies the difference between a pixel and its neighbor's intensities and ensures the smoothness of the lesion edges.

\subsubsection{Gaussian Kernel}

The Gaussian kernel is explored for Gaussian filtering [23]. The Gaussian kernel function is denoted as:

$$
k^{(m)}\left(f_{i}, f_{j}\right)=\exp \left(-\frac{1}{2}\left(f_{i}-f_{j}\right)^{T} \Lambda^{m}\left(f_{i}-f_{j}\right)\right)
$$

where $k^{(m)}\left(f_{i}, f_{j}\right)$ is the Gaussian Kernel function where vectors $f_{i}$ and $f_{j}$ are feature vectors for pixels $i$ and $j$ in an arbitrary feature space, and $\Lambda^{m}$ is a symmetric positive-definite precision matrix.

The pair-wise potentials can then be further be presented as:

$$
\psi\left(x_{i}, x_{j}\right)=\mu\left(x_{i}, x_{j}\right) \sum_{m=1}^{K} w^{(m)} k^{(m)}\left(f_{i}, f_{j}\right) .
$$

In the case of skin lesion images with color vectors, multi-class image segmentation is required with the color vectors $I_{i}$ and $I_{j}$. The process of multi-class segmentation is represented as:

$$
\begin{gathered}
k\left(f_{i}, f_{j}\right)=w^{(1)} \exp \left(-\frac{\left|p_{i}-p_{j}\right|^{2}}{2 \theta^{2}}-\frac{\left|I_{i}-I_{j}\right|^{2}}{2 \theta^{2}}\right)+m \\
m=w^{(2)} \exp \left(-\frac{\left|p_{i}-p_{j}\right|^{2}}{2 \theta^{2}} .\right.
\end{gathered}
$$

\subsubsection{Mean Field Approximation}

We achieved an efficient inference through an approximate inference program based on mean-field approximation. This reduces the variation free energy by computing a distribution $Q(x)$ instead of the exact distribution $P(x)$. This performs minimizing the KL-divergence $D(Q \| P)$, as shown in the equation below:

$$
Q(x)=N_{i} Q_{i}\left(X_{i}\right)
$$

This is the product of independent marginals $Q_{i}$ and $X_{i}$ respectively and $i$ ranges from 1 to N. Performing the sequential updates of $Q_{i}$ will guarantee the convergence.

The following have been achieved with the integration of the probabilistic model:

1. The probabilistic model, a CRF model, has performed the optimization of a single probability map through the use of local similarity of the neighborhood pixels;

2. In the model, pixels are captured through the unary potentials in the probability map generated and is further propagated through the neighborhood pixels captured in the pair-wise potentials;

3. The results generated at different stages are averaged and converged to produce the final integrated probability map;

4. Gaussian kernel is further utilized to smoothen the lesion boundary and fill holes; 
5. The utilization of the unary and pair-wise terms for extending the encoder-decoder network via a single feed-forward pass in the model has reasonably reduced the computational cost.

The algorithm for the proposed framework is depicted in Algorithm 1.

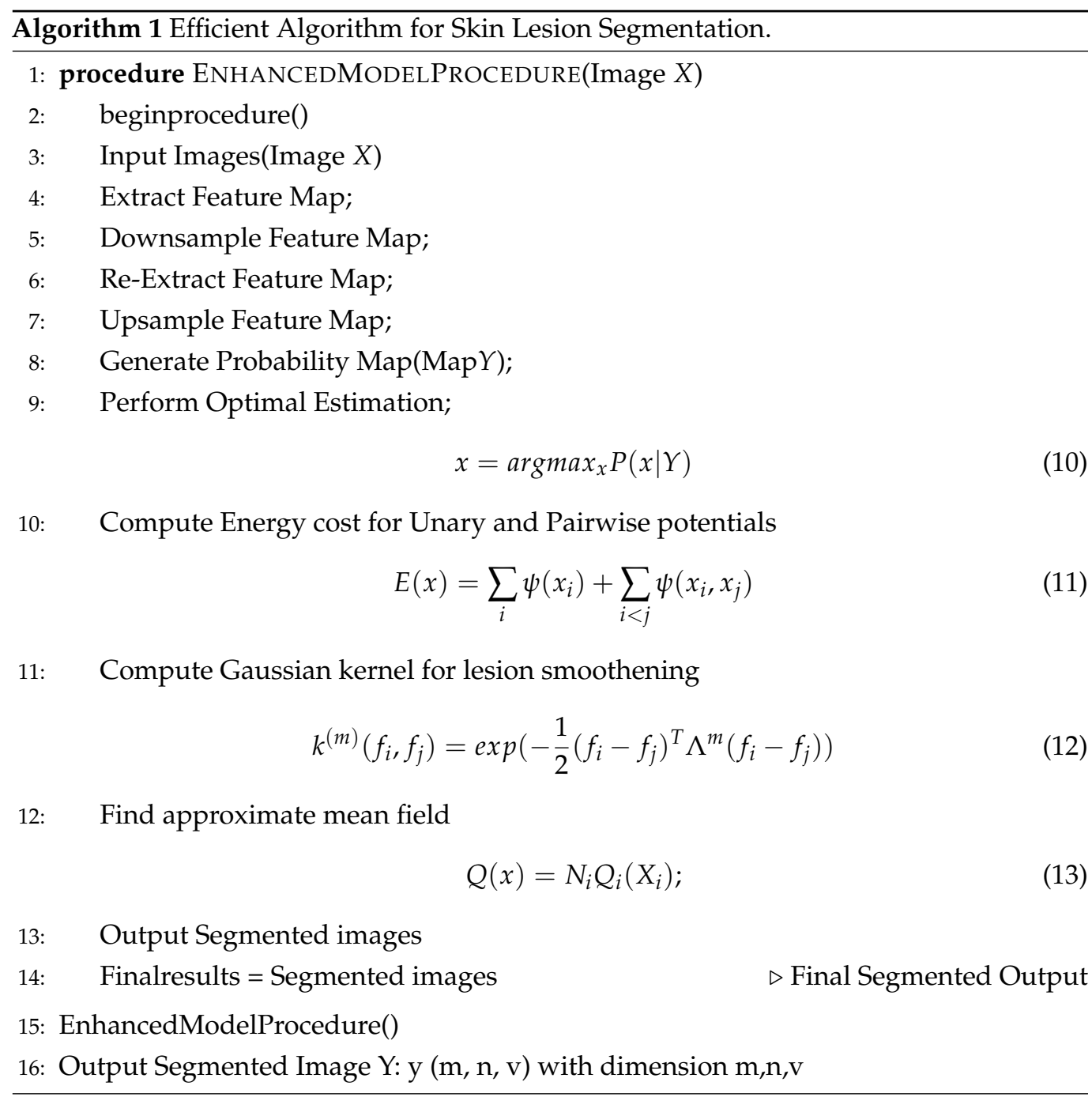

\subsection{Loss Function}

The loss function evaluates the level of uncertainty in this model. In this work, we employed the Dice loss function which computes the differences between the predicted probability and the actual results. This function computes the deviation of the predicted image pixels from the ground truth label pixels using the function stated below. The loss value, which represents the difference between the model's predicted probability distribution and the distribution of probabilities in the training dataset can then be used to represent the uncertainty.

$$
\text { DiceLoss }=1-\frac{2 \sum_{i} Y_{t} Y_{p}}{\sum_{i} Y_{t}^{2}+\sum_{i} Y_{p}^{2}}
$$

where $i$ is the pixels, $Y_{t}$ denotes the ground truth label, and $Y_{p}$ denotes the predicted image output. From the equation, the Dice loss function computes the losses for each class separately and then finds the average for the final score. 


\section{Experiments and Results}

In this section, various experiments were performed to evaluate the performance of each of the stages of our proposed framework. The experiment was actually performed several times over 500 times using a batch size of 32 and epoch number of 500 on the model. The evaluated results are displayed and explained below. The results are also compared with existing and similar approaches.

\subsection{Datasets}

The dataset used in this work is taken from ISBI 2017 [25] and divided into a training dataset composed of 2000 images, a validation dataset composed of 600 images, and testing dataset composed of 150 images. We also evaluated our proposed framework using a testing image dataset from PH2 [26] dataset. These datasets comprise both the images and ground truth labels with the images in a JPEG format while the ground truth are mask images in a PNG format.

\subsection{Results and Discussion}

The proposed framework has been evaluated and the results compared with the existing methods. First, the deep learning system is evaluated on the ISBI 2017 dataset without the enhancement in the first phase. The evaluation is based on the metrics such as accuracy, dice-coefficient, sensitivity, and specificity, respectively. In this phase, we achieved an accuracy of $96 \%$, a sensitivity of $98 \%$, and a dice coefficient of $92 \%$ and the specificity of $97 \%$ approximately as displayed in Table 1 . In the second phase, the deep learning system is enhanced with the probabilistic model with the Gaussian kernel. In this phase, the complete framework is also evaluated using the same metrics of accuracy, dice coefficient, sensitivity, and specificity, respectively on the ISBI dataset. We realized the improvements in the performance due to the enhancement with the new system producing a $97 \%$ accuracy, $98 \%$ sensitivity, and $94 \%$ dice coefficient and $98 \%$ specificity. This improvement is evident and clearly indicated in Figure 4.

Table 1. Performance analysis (\%) and comparison of the enhanced system with the method without an enhancement on the ISBI 2017 datatest.

\begin{tabular}{ccccc}
\hline Methods & Accuracy & Dice Score & Sensitivity & Spec \\
\hline Enhanced deep learning model & 97.0 & 94.0 & 98.0 & 98.0 \\
Deep learning model & 96.0 & 92.0 & 98 & 97 \\
\hline
\end{tabular}

The system computation time per image for the segmentation was also evaluated for the deep learning method and enhanced method. It is evident in the Table 2 that the deep learning method segmented a single testing image at $60 \mathrm{~s}$ while the enhanced method used an additional $10 \mathrm{~s}$ for the segmented results with the complete framework. This performance shows the effect of the model's light-weight size, which is composed of encoder-decoder network layers limited to just five levels with a minimized filter size for a lower consumption of computational resources.

Table 2. Proposed system performance evaluation based on processing time on the ISBI 2017.

\begin{tabular}{cc}
\hline Techniques & Time (s) \\
\hline Deep learning model & 60 \\
Enhanced deep learning model & 70 \\
\hline
\end{tabular}

The system also produces well-refined segmented skin lesion borders and as a result, the new framework can now subdue the effects of complex skin lesion with issues such as fuzzy and irregular edges and boundaries. The enhancement performed contour refinement and increased the accuracy and detection rate. It can be noticed from the seg- 
mentation results (Figure 5) that the enhanced segmentation method is able to detect the refined structures of the skin lesion edges and borders better than the fully convolutional deep learning system. The enhanced method utilized the Gaussian smoothening on the segmented images from the deep learning system to remove noise for better edge detection process. This can be observed in Table 3 where the system is evaluated using the Jaccard Index (where the area of overlap is calculated between the segmented binary image and the ground truth label) to predict the detection rate. The average Jaccard Index value of all the images were evaluated to $90.50 \%$ using the enhanced method, thus achieving a better performance than existing methods, as shown in Table 3. In addition, the system performance is displayed in the curves in Figures 6 and 7 for the system performance evaluation based on accuracy and dice coefficient metrics.

The improvement in the system performance also reflects when metrics such as dice coefficient and Jaccard Index are used for evaluation. This can also be seen in Table 3 where the system was evaluated using the Jaccard Index, only to predict the detection rate. The Jaccard Index and dice coefficient define the area of overlap that is calculated between the segmented binary image and ground truth label. The average Jaccard Index value of all the images evaluated to $90.50 \%$ using the enhanced method. This achieves a better performance than existing methods as shown in Table 3.

In addition, the results of the enhanced system was also compared with the existing methods as shown in Table 3 using the Jaccard Index and Table 4, using metrics such as accuracy, dice-coefficient, sensitivity, and specificity. In the two tables, the enhanced system outperforms the existing state-of-the-arts in all the evaluation metrics. This shows that the proposed system is more reliable and efficient than some of the existing systems.

Some pretrained model have been used for the benchmark of the proposed model. The pretrained models have been evaluated on the ISBI 2017 dataset and their performance evaluated against the proposed model. The proposed model outperforms the pretrained model as shown in Table 5. Lastly, the proposed model was evaluated on the PH2 dataset and the results are shown in Table 6 for the deep learning model and enhanced model. Lastly, the proposed system utilizes a light-weight framework with a reduced number of parameters when compared with existing methods as shown in Table 7. This also minimizes the computational resources requirements.

Table 3. Performance analysis (\%) based on the Jaccard Index for only the proposed method with existing probability-based methods on the ISBI 2017.

\begin{tabular}{cc}
\hline Methods & Jaccard Index (JI) \\
\hline Our Method & 90.50 \\
CUNET [27] & 75.5 \\
DCNNCRF [28] & 80.2 \\
YOLO [29] & 74.81 \\
\hline
\end{tabular}

Table 4. Performance analysis (\%) and comparison of the proposed method with existing probability based methods on the ISBI 2017 datatest.

\begin{tabular}{ccccc}
\hline Methods & Accuracy & Dice Score & Sensitivity & Spec \\
\hline Proposed enhanced system & 97.0 & 94.0 & 98.0 & 98.0 \\
CUNET [27] & 90.04 & 87.49 & 47.32 & - \\
YOLO [29] & 93.39 & 84.26 & 90.82 & 92.68 \\
GAUSIAN [30] & 93.6 & 91.1 & 100 & 92.5 \\
MDRFI [21] & 94.3 & 90.7 & - & - \\
FCNCRF [31] & 97.8 & - & 95.1 & 98.0 \\
\hline
\end{tabular}


Table 5. Performance analysis (\%) and comparison of the proposed method with existing pre-trained models on the ISBI 2017 datatest.

\begin{tabular}{ccccc}
\hline Methods & Accuracy & Dice Score & Sensitivity & Spec \\
\hline Proposed enhanced system & 97.0 & 94.0 & 98.0 & 98.0 \\
deeplabv3 [32] & 94.3 & 85.8 & 83.5 & 98.2 \\
DenseNet169 [32] & 93.8 & 86.8 & 86.8 & 96.3 \\
ResNet152 [32] & 94.1 & 87.1 & 87.7 & 96.1 \\
Xception [32] & 94.4 & 87.4 & 86.4 & 97.1 \\
ResNetV2 [32] & 94.4 & 87.6 & 87.3 & 96.4 \\
Efficientnet [33,34] & 96.7 & 87.2 & 54.5 & 96.4 \\
\hline
\end{tabular}

Table 6. Performance analysis (\%) and comparison of the enhanced system with the method without enhancement on the $\mathrm{PH} 2$ datatest.

\begin{tabular}{ccccc}
\hline Methods & Accuracy & Dice Score & Sensitivity & Spec \\
\hline Enhanced deep learning model & 96.0 & 93.0 & 94.0 & 96.0 \\
Deep learning model & 95.0 & 92.0 & 93 & 95 \\
\hline
\end{tabular}

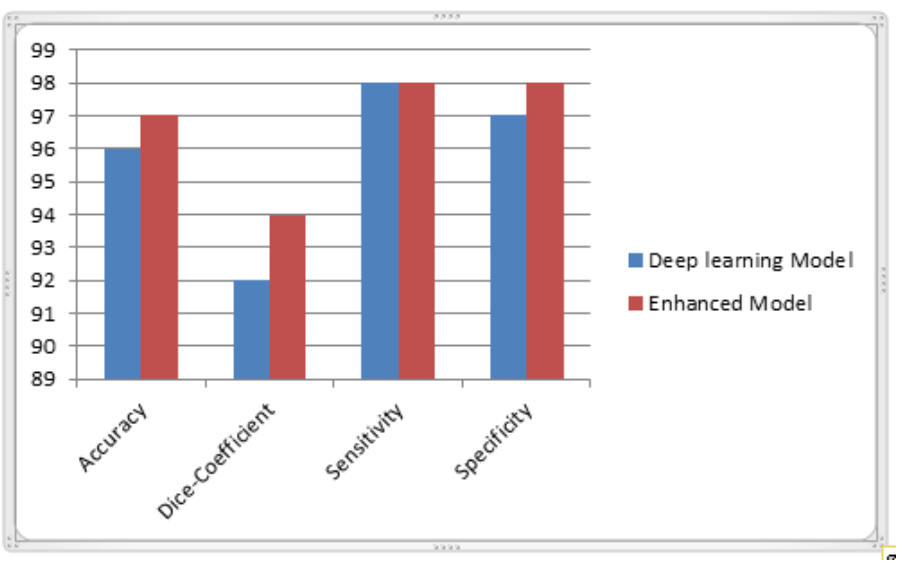

Figure 4. The figure shows the performance of the deep learning model against the enhanced method on ISBI 2017 skin lesion images .

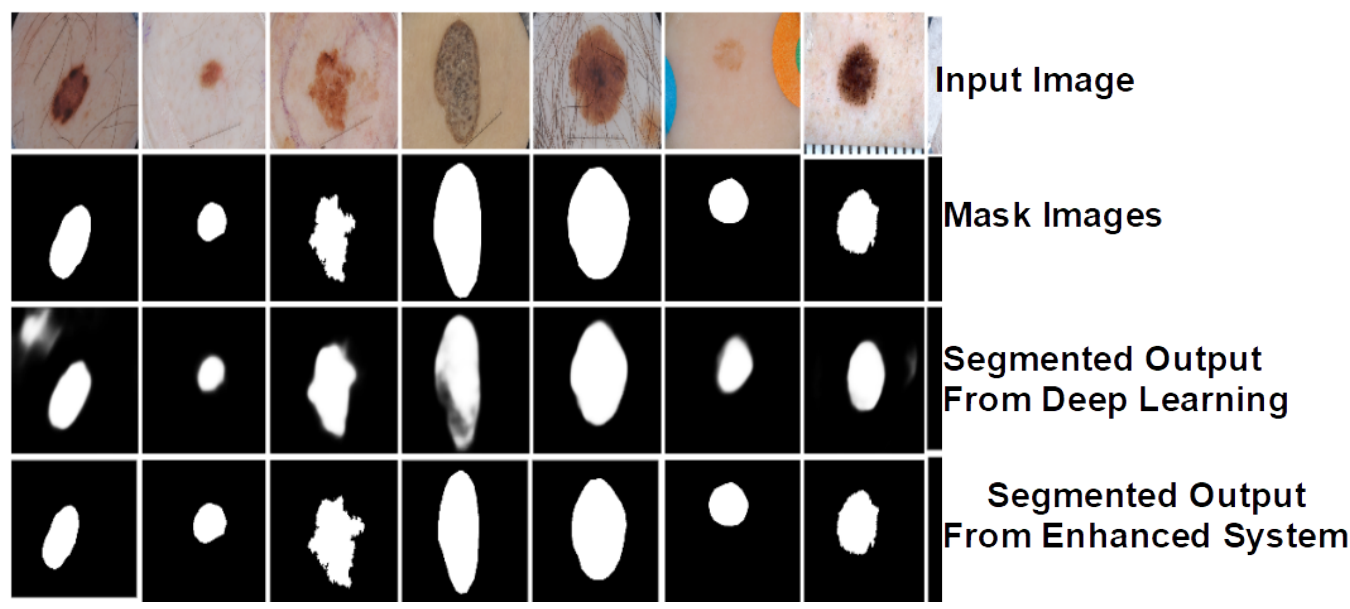

Figure 5. The figure shows the segmentation outcomes of the system on the ISBI 2017 dataset: The first row represents the input images; the second rows represents the ground truth labels; the third row represent segmented results on the deep learning model; and the last row shows the segmented results of the enhanced system. 


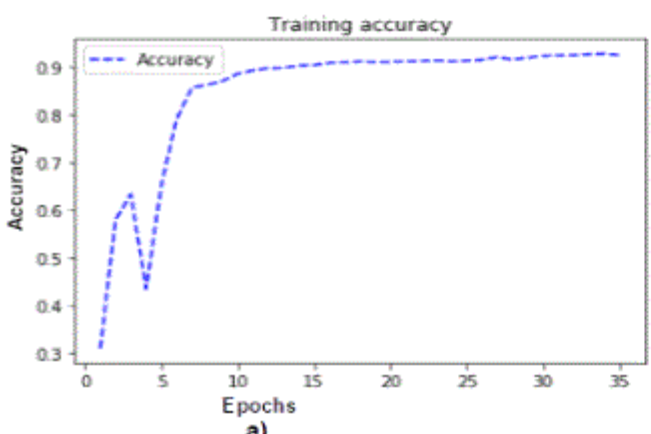

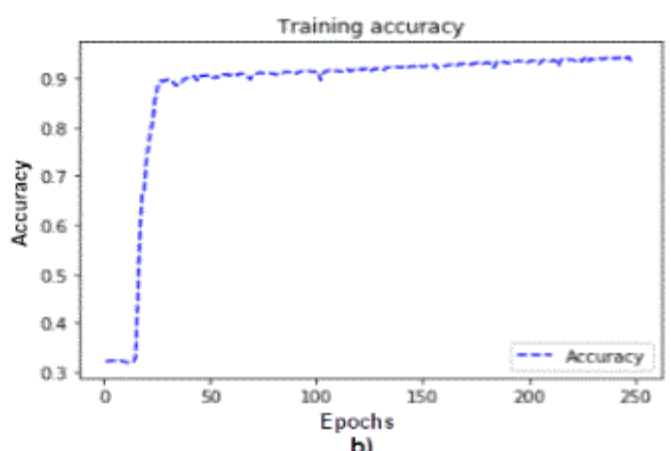

b)

Figure 6. The training accuracy results curves of the proposed system on the ISBI 2017 and PH2 datasets. (a) Accuracy results curve on ISBI 2017 dataset; (b) Accuracy results curve on PH2 dataset.
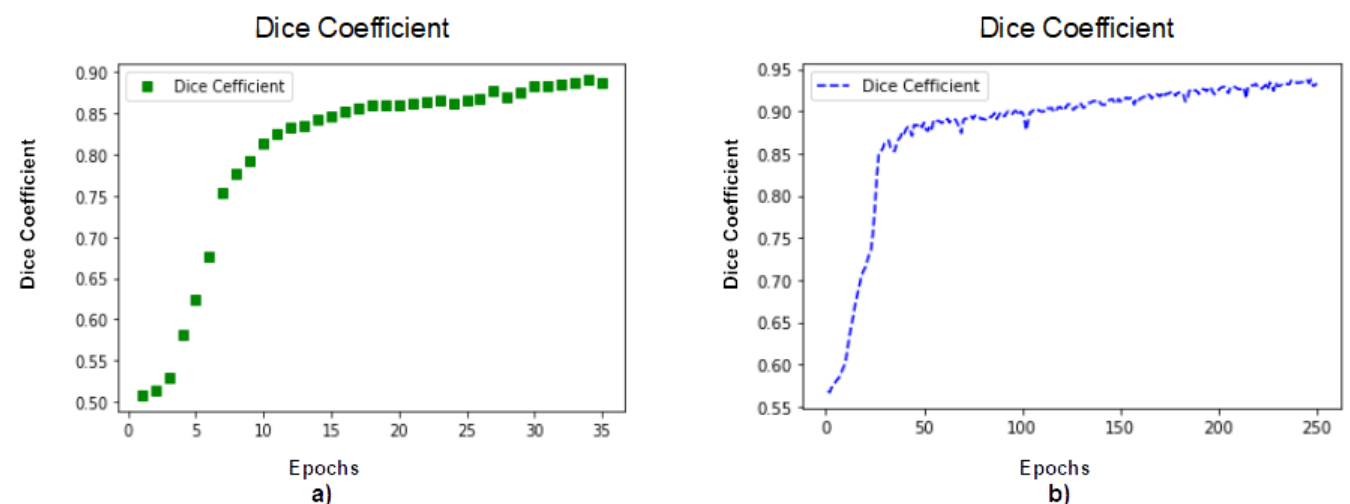

b)

Figure 7. Dice coefficient results curves of the proposed system on the ISBI 2017 and PH2 datasets. (a) Dice coefficient results curve on ISBI 2017 dataset; (b) Dice coefficient results curve on PH2 dataset.

Figure 8 also shows that the training loss diminishes to less than $10 \%$ as the training accuracy increases. This figure shows a direct relationship between the loss rate and the uncertainty in segmentation prediction from the ground truth labels. Any reasonable reduction in the loss rate also indicates an improved performance and will improve the performance of the proposed model.
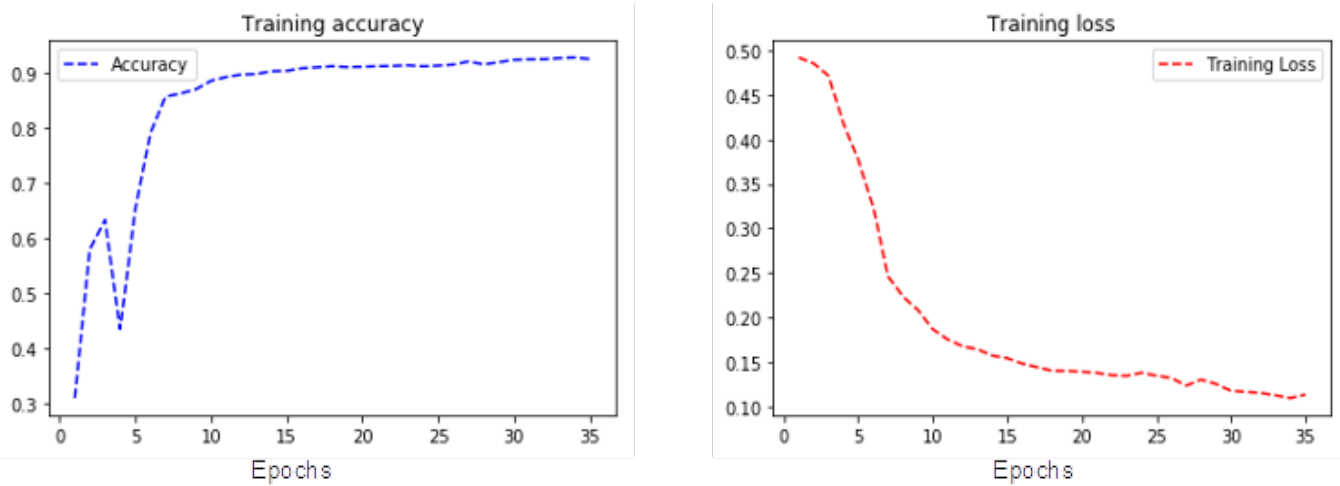

Figure 8. This displays two figures showing the training accuracy and loss curve respectively for the training of the proposed model on ISBI 2017. 
Table 7. Proposed system performance evaluation based on number of parameters, loss function and mIOU on ISBI 2017.

\begin{tabular}{cccc}
\hline Techniques & Param (Number of Parameters) & Loss Function & mIOU \\
\hline FCN8 [35] & $138 \mathrm{M}$ & Cross Entropy with IOU & 70.7 \\
UNET [35] & $38 \mathrm{M}$ & Cross Entropy with IOU & 73.9 \\
DSNET [35] & $10 \mathrm{M}$ & Cross Entropy with IOU & 77.5 \\
FCN-based DenseNet [24] & $11.5 \mathrm{M}$ & DiceLoss & 93 \\
PROPOSED & $6.97 \mathrm{M}$ & DiceLoss & 94 \\
\hline
\end{tabular}

\section{Conclusions}

Conclusively, an efficient and reliable framework with enhanced deep learning model was proposed for the analysis and segmentation of skin lesion images. The system was able to overcome the challenges with irregular and fuzzy skin lesion borders. The probabilistic model (CRF) utilized Gaussian Edge Potentials with mean-field approximation for smoothening the weak edges, as well as the irregular and fuzzy borders of skin lesion images. The deep learning system utilized a light-weight fully convolutional deep learning system. The framework tested on publicly available skin lesion images dataset achieved significant accuracy. The system was also compared with existing state-of-the-arts techniques using metrics such as the Jaccard Index, sensitivity, and specificity achieving a better performance than the existing system.

Author Contributions: The individual contributions are provided as follows where A.A.A. represents Adekanmi Adeyinka Adegun, S.V. represents Serestina Viriri and M.H.Y. represents Muhammad Haroon Yousaf: Conceptualization, A.A.A. and S.V.; methodology, A.A.A. and S.V.; software, A.A.A.; validation, A.A.A., S.V. and M.H.Y.; formal analysis, S.V.; investiga-tion, S.V.; resources, S.V.; data curation, A.A.A.; writing — original draft preparation, A.A.A.; writ-ing—review and editing, S.V. and M.H.Y.; visualization, S.V.; supervision, S.V.; project administration, S.V.; funding acquisition, S.V. All authors have read and agreed to the published version of the manuscript.

Funding: This research received no external funding.

Informed Consent Statement: Informed consent was obtained from all subjects involved in the study.

Data Availability Statement: Data available in a publicly accessible repository: The data presented in this study are openly available in ISBI and PH2.

Conflicts of Interest: The authors declare no conflict of interest.

\section{References}

1. Prasad, R.R.; Paudel, S.; Raina, K.; Agarwal, R. Silibinin and non-melanoma skin cancers. J. Tradit. Complement. Med. 2020. [CrossRef] [PubMed]

2. Halpern, A.C.; Marghoob, A.A.; Reiter, O. Melanoma Overview: Skin Cancer Foundation. April 2019. Available online: https://www.skincancer.org/skin-cancer-information/melanoma (accessed on 31 December 2020).

3. World Health Organization. Ultraviolet (UV) Radiation and Skin Cancer. October 2017. Available online: https://www.who.int/ news-room/q-a-detail/ultraviolet-(uv)-radiation-and-skin-cancer (accessed on 31 December 2020 ).

4. Ngoc-Quang, N.; Lee, S. Robust Boundary Segmentation in Medical Images using a Consecutive Deep Encoder-Decoder Network. IEEE Access 2019, 7, 33795-33807.

5. Al-Masni, M.A.; Al-antari, M.A.; Choi, M.; Han, S.; Kim, T. Skin lesion segmentation in dermoscopy images via deep full resolution convolutional networks. Comput. Methods Programs Biomed. 2018, 162, 221-231. [CrossRef] [PubMed]

6. Silveira, M.; Nascimento, J.C.; Marques, J.S.; Marcal, A.R.S.; Mendonca, T.; Yamauchi, S.; Maeda, J.; Rozeira, J. Comparison of segmentation methods for melanoma diagnosis in dermoscopy images. IEEE J. Sel. Top. Signal Process. 2009, 3, 35-45. [CrossRef]

7. Celebi, E.; Wen, M.Q.; Hwang, S.; Iyatomi, H.; Schaefer, G. Lesion border detection in dermoscopy images using ensembles of thresholding methods. Ski. Res. Technol. 2013, 19, e252-e258. [CrossRef] [PubMed]

8. Kajsa, M.; Kirchesch, H.M.; Schopf, T.G.; Godtliebsen, F. Unsupervised segmentation for digital dermoscopic images. Ski. Res. Technol. 2010, 16, 401-407.

9. Francesco, P.; Bogo, F.; Bonazza, M.; Cappelleri, V.; Peserico, E. Simpler, faster, more accurate melanocytic lesion segmentation through meds. IEEE Trans. Biomed. Eng. 2013, 61, 557-565.

10. Emin, Y.M.; Borlu, M. Accurate segmentation of dermoscopic images by image thresholding based on type-2 fuzzy logic. IEEE Trans. Fuzzy Syst. 2009, 17, 976-982. 
11. Fengying, X.; Bovik, A.C. Automatic segmentation of dermoscopy images using self-generating neural networks seeded by genetic algorithm. Pattern Recognit. 2013, 46, 1012-1019.

12. Masood, A.; Al-Jumaily, A.A. Computer aided diagnostic support system for skin cancer: A review of techniques and algorithms. Int. J. Biomed. Imaging 2013. [CrossRef]

13. Zhu, W.; Huang, Y.; Zeng, L.; Chen, X.; Liu, Y.; Qian, Z.; Du, N.; Fan, W.; Xie, X. AnatomyNet: Deep learning for fast and fully automated whole-volume segmentation of head and neck anatomy. Med. Phys. 2019, 46, 576-589. [CrossRef] [PubMed]

14. Andre, E.; Kuprel, B.; Novoa, R.A.; Ko, J.; Swetter, S.M.; Blauet, H.M. Dermatologist-level classication of skin cancer with deep neural networks. Nature 2017, 542, 115.

15. Baig, R.; Bibi, M.; Hamid, A.; Kausar, S.; Khalid, S. Deep Learning Approaches Towards Skin Lesion Segmentation and Classification from Dermoscopic Images-A Review. Curr. Med Imaging 2020, 16, 513-533. [CrossRef]

16. Khan, A.M.; Akram, T.; Sharif, M.; Javed, K.; Rashid, M.; Bukhari, S.A.C. An integrated framework of skin lesion detection and recognition through saliency method and optimal deep neural network features selection. Neural Comput. Appl. 2019, 32, 15929-15948. [CrossRef]

17. Sigurdsson, S.; Philipsen, P.A.; Hansen, L.K.; Larsen, J.; Gniadecka, M.; Wulf, H. Detection of skin cancer by classification of Raman spectra. IEEE Trans. Biomed. Eng. 2004, 51, 1784-1793. [CrossRef] [PubMed]

18. Barata, C.; Ruela, M.; Mendonça, T.; Marques, J.S. A bag-of-features approach for the classification of melanomas in dermoscopy images: The role of color and texture descriptors. In Computer Vision Techniques for the Diagnosis of Skin Cancer; Springer: Berlin/Heidelberg, Germany, 2014; pp. 49-69.

19. Ahn, E.; Kim, J.; Bi, L.; Kumar, A.; Li, C.; Fulham, M.; Feng, D.D. Saliency-based lesion segmentation via background detection in dermoscopic images. IEEE J. Biomed. Health Inform. 2017, 21, 1685-1693. [CrossRef]

20. Bi, L.; Kim, J.; Ahn, E.; Kumar, A.; Feng, D.; Fulham, M. Step-wise integration of deep class-specific learning for dermoscopic image segmentation. Pattern Recognit. 2019, 85, 78-89. [CrossRef]

21. Jahanifar, M.; Tajeddin, N.Z.; Asl, B.M.; Gooya, A. Supervised saliency map driven segmentation of lesions in dermoscopic images. IEEE J. Biomed. Health Inform. 2018, 23, 509-518. [CrossRef]

22. Adegun, A.; Viriri, S. An enhanced deep learning framework for skin lesions segmentation. In Proceedings of the International Conference on Computational Collective Intelligence, Hendaye, France, 4-6 September 2019; pp. 414-425.

23. Philipp, K.; Koltun, V. Efficient inference in fully connected crfs with gaussian edge potentials. In Advances in Neural Information Processing Systems; MIT Press: Cambridge, MA, USA, 2011; pp. 109-117.

24. Adegun, A.A.; Viriri, S. FCN-Based DenseNet Framework for Automated Detection and Classification of Skin Lesions in Dermoscopy Images. IEEE Access 2020, 8, 150377-150396. [CrossRef]

25. Codella, N.C.F.; Gutman, D.; Celebi, M.E.; Helba, B.; Marchetti, M.A.; Dusza, S.W.; Kalloo, A.; Liopyris, K.; Mishra, N.; Kittler, H.; et al. Skin lesion analysis toward melanoma detection: A challenge at the 2017 international symposium on biomedical imaging (isbi), hosted by the international skin imaging collaboration (isic). In Proceedings of the 2018 IEEE 15th International Symposium on Biomedical Imaging (ISBI 2018), Washington, DC, USA, 4-7 April 2018; pp. 168-172.

26. Teresa, M.; Ferreira, P.M.; Marques, J.S.; Marcal, A.R.; Rozeira, J. PH 2-A dermoscopic image database for research and benchmarking. In Proceedings of the 2013 35th Annual International Conference of the IEEE Engineering in Medicine and Biology Society (EMBC), Osaka, Japan, 3-7 July 2013; pp. 5437-5440.

27. Jiang, H.; Rong, R.; Wu, J.; Li, X.; Dong, X.; Chen, E.Z. Skin lesion segmentation with improved C-UNet networks. BioRxiv 2018, 382549. [CrossRef]

28. Qiu, Y.; Cai, J.; Qin, X.; Zhang, J. Inferring Skin Lesion Segmentation With Fully Connected CRFs Based on Multiple Deep Convolutional Neural Networks. IEEE Access 2020, 8, 144246-144258. [CrossRef]

29. Ünver, M.H.; Ayan, E. Skin lesion segmentation in dermoscopic images with combination of YOLO and grabcut algorithm. Diagnostics 2019, 9, 72. [CrossRef] [PubMed]

30. Ali, A.-R.; Li, J.; Yang, G.; O'Shea, S.J. A machine learning approach to automatic detection of irregularity in skin lesion border using dermoscopic images. Peerj Comput. Sci. 2020, 6, e268. [CrossRef]

31. Luo, W.; Yang, M. Fast skin lesion segmentation via fully convolutional network with residual architecture and CRF. In Proceedings of the 2018 24th International Conference on Pattern Recognition (ICPR), Beijing, China, 20-24 August 2018; pp. 1438-1443.

32. Jahanifar, M.; Tajeddin, N.Z.; Koohbanani, N.A.; Gooya, A.; Rajpoot, N. Segmentation of skin lesions and their attributes using multi-scale convolutional neural networks and domain specific augmentations. arXiv 2018, arXiv:1809.10243.

33. Gessert, N.; Nielsen, M.; Shaikh, M.; Werner, R.; Schlaefer, A. Skin lesion classification using ensembles of multi-resolution EfficientNets with meta data. MethodsX 2020, 100864. [CrossRef]

34. Rocheteau, E.; Kim, D. Deep Transfer Learning for Automated Diagnosis of Skin Lesions from Photographs. arXiv 2020, arXiv:2011.04475.

35. Hasan, K.M.; Dahal, L.; Samarakoon, P.N.; Tushar, F.I.; Martí, R. DSNet: Automatic dermoscopic skin lesion segmentation. Comput. Biol. Med. 2020, 103738. [CrossRef] 